\title{
GENERALIZED SYMMETRIC SPACES, YU. P. SOLOVYOV'S FORMULA, AND THE GENERALIZED HERMITIAN GEOMETRY
}

\author{
V. V. Balashchenko
}

UDC 514.765

Dedicated to the memory of Yu. P. Solovyov

\begin{abstract}
We collect some basic results on canonical affinor structures of classical type on generalized symmetric spaces. Yu. P. Solovyov's stimulating influence on this topic during its initial stages is illustrated. Using special canonical $f$-structures on homogeneous $k$-symmetric spaces, we also present a new collection of homogeneous Hermitian $f$-manifolds.
\end{abstract}

\section{Introduction}

An important place among homogeneous manifolds of Lie groups is occupied by homogeneous $\Phi$-spaces, i.e., homogeneous spaces generated by automorphisms $\Phi$ of Lie groups (see $[15,21,30,44,54,58]$ ). A special role is played by regular $\Phi$-spaces, which were first introduced in [54]. As is known [54], such $\Phi$-spaces include all homogeneous $k$-symmetric spaces $\left(\Phi^{k}=\mathrm{id}\right)[44]$ and are contained, in turn, in the well-known class of homogeneous reductive spaces. At the same time, a distinguishing feature of regular $\Phi$-spaces is that each such space has a natural associated object, the commutative algebra $\mathcal{A}(\theta)$ of canonical affinor structures [15]. This algebra contains well-known classical structures, such as almost complex structures, almost product structures, $f$-structures of K. Yano $\left(f^{3}+f=0\right)$, and $h$-structures $\left(h^{3}-h=0\right)($ see $[3,15])$. The first example in this direction was the canonical almost complex structure on homogeneous 3-symmetric spaces (see $[24,55,58]$ ). It should be mentioned that this structure was effectively applied to many constructions in differential geometry and global analysis (see Sec. 2). We especially stress that the canonical almost complex structure $J$ on homogeneous 3 -symmetric spaces provided a remarkable class of invariant examples in the theory of almost Hermitian structures, in particular, nearly Kähler structures (see $[24,35,58]$ and many others). It turns out that canonical $f$-structures on homogeneous $k$-symmetric spaces play a similar role for the generalized Hermitian geometry created in the early 1980s (see, e.g., [38,39]) and intensively studied in differential geometry.

The paper is organized as follows.

In Secs. 2-4, we collect basic notions and results on homogeneous regular $\Phi$-spaces, specifically, homogeneous $k$-symmetric spaces. First encouraging examples of canonical structures are discussed and Yu. P. Solovyov's stimulating influence on this direction during its first stage is illustrated. Moreover, a precise description of all canonical structures of classical type on homogeneous $k$-symmetric spaces is included.

In Secs. 5 and 6 , we consider special canonical $f$-structures on regular $\Phi$-spaces and establish an important property for invariant distributions generated by these $f$-structures. We also briefly select basic notions in the generalized Hermitian geometry, including principal classes of metric $f$-structures. The main result is that the special canonical $f$-structures on homogeneous $k$-symmetric spaces $(k=4 n$, $n \geq 1$ ) present a new collection of invariant Hermitian $f$-structures. Some particular cases are discussed in more detail.

\section{Homogeneous Regular $\Phi$-Spaces}

Here we briefly formulate some basic definitions and results related to regular $\Phi$-spaces and selected canonical structures on them. More detailed information can be found in [11, 15, 21, 44, 54, 55, 58].

Translated from Fundamentalnaya i Prikladnaya Matematika, Vol. 13, No. 8, pp. 43-60, 2007. 
Let $G$ be a connected Lie group and $\Phi$ be an (analytic) automorphism of it. Denote by $G^{\Phi}$ the subgroup of all fixed points of $\Phi$ and by $G_{o}^{\Phi}$ the identity component of $G^{\Phi}$. Assume that a closed subgroup $H$ of $G$ satisfies the condition

$$
G_{o}^{\Phi} \subset H \subset G^{\Phi} .
$$

Then $G / H$ is called a homogeneous $\Phi$-space.

Homogeneous $\Phi$-spaces include homogeneous symmetric spaces $\left(\Phi^{2}=\mathrm{id}\right)$ and, more generally, $h_{o}$ mogeneous $\Phi$-spaces of order $k\left(\Phi^{k}=\mathrm{id}\right)$ or, in other terminology, homogeneous $k$-symmetric spaces (see [44]).

For any homogeneous $\Phi$-space $G / H$, one can define the mapping

$$
S_{o}=D: G / H \rightarrow G / H, \quad x H \rightarrow \Phi(x) H .
$$

It is known [54] that $S_{o}$ is an analytic diffeomorphism of $G / H . S_{o}$ is usually called a "symmetry" of $G / H$ at the point $o=H$. It is obvious that in view of the homogeneity, the "symmetry" $S_{p}$ can be defined at any point $p \in G / H$. More exactly, for any $p=\tau(x) o=x H, q=\tau(y) o=y H$ we set

$$
S_{p}=\tau(x) \circ S_{o} \circ \tau\left(x^{-1}\right) .
$$

It is easy to show that

$$
S_{p}(y H)=x \Phi\left(x^{-1}\right) \Phi(y) H .
$$

Thus, any homogeneous $\Phi$-space is equipped with the set of symmetries $\left\{S_{p} \mid p \in G / H\right\}$. Moreover, each $S_{p}$ is an analytic diffeomorphism of the manifold $G / H$ (see [54]).

Note that there exist homogeneous $\Phi$-spaces that are not reductive. Hence, the so-called regular $\Phi$-spaces first introduced by N. A. Stepanov [54] are of fundamental importance.

Let $G / H$ be a homogeneous $\Phi$-space, $\mathfrak{g}$ and $\mathfrak{h}$ be the corresponding Lie algebras for $G$ and $H$, and $\varphi=d \Phi_{e}$ the automorphism of $\mathfrak{g}$. Consider the linear operator $A=\varphi-$ id and the Fitting decomposition $\mathfrak{g}=\mathfrak{g}_{0} \oplus \mathfrak{g}_{1}$ with respect to $A$, where $\mathfrak{g}_{0}$ and $\mathfrak{g}_{1}$ denote the 0 - and 1-components of the decomposition, respectively. Further, let $\varphi=\varphi_{s} \varphi_{u}$ be the Jordan decomposition, where $\varphi_{s}$ and $\varphi_{u}$ are the semisimple and unipotent components of $\varphi$, respectively, $\varphi_{s} \varphi_{u}=\varphi_{u} \varphi_{s}$. Denote by $\mathfrak{g}^{\gamma}$ the subspace of all fixed points for a linear endomorphism $\gamma$ in $\mathfrak{g}$. It is clear that $\mathfrak{h}=\mathfrak{g}^{\varphi}=\operatorname{Ker} A, \mathfrak{h} \subset \mathfrak{g}_{0}$, and $\mathfrak{h} \subset \mathfrak{g}^{\varphi_{s}}$.

Definition 1 ([11, 15,21,54]). A homogeneous $\Phi$-space $G / H$ is called a regular $\Phi$-space if one of the following equivalent conditions is satisfied:

(1) $\mathfrak{h}=\mathfrak{g}_{0}$

(2) $\mathfrak{g}=\mathfrak{h} \oplus A \mathfrak{g}$;

(3) the restriction of the operator $A$ to $A \mathfrak{g}$ is nonsingular;

(4) $A^{2} X=0 \Longrightarrow A X=0$ for all $X \in \mathfrak{g}$;

(5) the matrix of the automorphism $\varphi$ can be represented in the form

$$
\left(\begin{array}{cc}
E & 0 \\
0 & B
\end{array}\right)
$$

where the matrix $B$ does not admit the eigenvalue 1 ;

(6) $\mathfrak{h}=\mathfrak{g}^{\varphi_{s}}$.

We recall two basic facts.

Theorem 1 ([54]).

(1) Any homogeneous $\Phi$-space of order $k\left(\Phi^{k}=\mathrm{id}\right)$ is a regular $\Phi$-space.

(2) Any regular $\Phi$-space is reductive. More exactly, the Fitting decomposition

$$
\mathfrak{g}=\mathfrak{h} \oplus \mathfrak{m}, \quad \mathfrak{m}=A \mathfrak{g},
$$

is a reductive one. 
Decomposition (1) is the canonical reductive decomposition corresponding to a regular $\Phi$-space $G / H$, and $\mathfrak{m}$ is the canonical reductive complement.

We also note that for any regular $\Phi$-space $G / H$, each point $p=x H \in G / H$ is an isolated fixed point of the symmetry $S_{p}$ (see [54]).

Decomposition (1) is obviously $\varphi$-invariant. Denote by $\theta$ the restriction of $\varphi$ to $\mathfrak{m}$. As usual, we identify $\mathfrak{m}$ with the tangent space $T_{o}(G / H)$ at the point $o=H$. It is important to note that the operator $\theta$ commutes with any element of the linear isotropy group $\operatorname{Ad}(H)$ (see [54]). It also should be noted (see [54]) that

$$
\left(d S_{o}\right)_{o}=\theta \text {. }
$$

Now we turn to the remarkable invariant structures on homogeneous $k$-symmetric spaces. Note that for any homogeneous $k$-symmetric space $G / H$, the following identity holds:

$$
\theta^{k-1}+\theta^{k-2}+\cdots+\theta+\mathrm{id}=0 .
$$

It was discovered in the late 1960s that an arbitrary homogeneous 3 -symmetric space $G / H$ admits the canonical almost complex structure $J$ (see $[55,58]$ ). This structure was defined by the linear endomorphism

$$
J_{o}=\frac{1}{\sqrt{3}}\left(\theta^{2}-\theta\right)
$$

on $\mathfrak{m}$ because $J_{o}^{2}=-\mathrm{id}$ (see [55]). This implies that the structure $J$ is invariant with respect to the Lie group $G$ as well as the symmetries $S_{p}$. It should be mentioned that the canonical almost complex structure on such spaces became an effective tool and a remarkable example in many constructions of differential geometry and global analysis such as almost Hermitian structures (see $[24,25,35,58]$ ), homogeneous structures (see $[1,22,37,46,50,57]$, etc.), Einstein metrics (see $[51,52])$, holomorphic and minimal submanifolds (see $[48,49]$ ), and real Killing spinors (see $[17,29,33]$ ).

In 1990, another structure of such a kind was presented. It was the canonical almost product structure $P$ on any homogeneous 5 -symmetric space $G / H$ defined by the formula (see [12])

$$
P_{o}=\frac{1}{\sqrt{5}}\left(\theta^{4}-\theta^{3}-\theta^{2}+\theta\right) \text {. }
$$

It gave the opportunity to obtain a number of general results on these spaces (see $[12,18-20]$ ).

\section{Solovyov's Formula}

Taking into account the formulas for the canonical structures $J$ and $P$ on homogeneous 3 - and 5 -symmetric spaces, respectively (see the previous section), Solovyov proposed their extension for an arbitrary homogeneous $p$-symmetric space, where $p$ is a prime odd number. In this case, the situation can be described in a purely algebraic way.

Proposition 1. The cyclotomic field $\mathbb{Q}\left(\varepsilon_{p}\right)$ contains the square root of the element $p *=(-1)^{\frac{p-1}{2}} p$, where $p$ is a prime odd number.

Proof (1990, originally handwritten by Solovyov). We obviously have

$$
p=\prod_{i=1}^{p-1}\left(1-\varepsilon_{p}^{i}\right) .
$$

Combining the factors corresponding to $i$ and $p-i$, we obtain

$$
\left(1-\varepsilon_{p}^{i}\right)\left(1-\varepsilon_{p}^{p-i}\right)=\left(1-\varepsilon_{p}^{i}\right)\left(1-\varepsilon_{p}^{-i}\right)=-\varepsilon_{p}^{-i}\left(1-\varepsilon_{p}^{i}\right)^{2} .
$$

It follows that

$$
p=(-1)^{\frac{p-1}{2}} \varepsilon_{p}^{b} \prod_{i=1}^{\frac{p-1}{2}}\left(1-\varepsilon_{p}^{i}\right)^{2}
$$


where $b=-(1+2+\cdots+(p-1) / 2)$. Choosing $c \in \mathbb{Z}$ such that $2 c \equiv 1(\bmod p)$, we obtain $\varepsilon_{p}^{b}=\left(\varepsilon_{p}^{b c}\right)^{2}$. This means that the previous formula can be written as follows:

$$
p=(-1)^{\frac{p-1}{2}}\left(\varepsilon_{p}^{b c}\right)^{2} \prod_{i=1}^{\frac{p-1}{2}}\left(1-\varepsilon_{p}^{i}\right)^{2} .
$$

Finally, we obtain

$$
p *=(-1)^{\frac{p-1}{2}} p=\left(\varepsilon_{p}^{b c} \prod_{i=1}^{\frac{p-1}{2}}\left(1-\varepsilon_{p}^{i}\right)\right)^{2}
$$

This completes the proof.

As an immediate application, we have the following corollary.

Corollary 1. Let $G / H$ be a homogeneous $p$-symmetric space, where $p$ is a prime odd number. Then $G / H$ admits

the canonical almost complex structure $J$ if $p \equiv 3(\bmod 4)$;

the canonical almost product structure $P$ if $p \equiv 1(\bmod 4)$.

Note that formula (2) gives the algorithm for calculating the polynomials in $\theta$ for the canonical structures $J$ and $P$ above mentioned. Moreover, the formula for these structures can be indicated in the explicit form:

$$
F(\theta)=\frac{1}{\sqrt{p}}\left(\theta+\theta^{4}+\cdots+\theta^{p^{2}}\right) .
$$

In particular, for $p=3$ and $p=5$, we obtain (up to sign) the structures $J$ and $P$ (see Sec. 2). As an example, for $p=7$ we obtain

$$
J=\frac{1}{\sqrt{7}}\left(\theta+\theta^{2}-\theta^{3}+\theta^{4}-\theta^{5}-\theta^{6}\right) .
$$

Remark 1. It should be mentioned that the assertion of Proposition 1 is well known (see, e.g., [45, Chap. 8]). Nevertheless, the proof indicated above is fairly original and simple. In addition, as we already noted, it provides an easy method for explicitly calculating $\sqrt{p *}$.

\section{Canonical Affinor Structures on Regular $\Phi$-Spaces}

In 1990, being encouraged by Solovyov's interest in the problem and under his stimulating influence, we tried to observe the situation in general. It turned out that, using a completely different technique, it was possible to describe all canonical classical structures for any homogeneous regular $\Phi$-space. Moreover, for all homogeneous $k$-symmetric spaces, precise computational formulas for these structures were indicated. This general approach together with the main results is briefly presented here.

An affinor structure on a manifold is known to be a tensor field of type $(1,1)$ or, equivalently, a field of endomorphisms acting on its tangent bundle. Assume that $F$ is an invariant affinor structure on a homogeneous manifold $G / H$. Then $F$ is completely determined by its value $F_{o}$ at the point $o$, where $F_{o}$ is invariant with respect to $\operatorname{Ad}(H)$. For simplicity, we denote in the same manner both any invariant structure on $G / H$ and its value at $o$ throughout the rest of the paper.

Definition $2([14,15])$. An invariant affinor structure $F$ on a regular $\Phi$-space $G / H$ is called canonical if its value at the point $o=H$ is a polynomial in $\theta$.

Denote by $\mathcal{A}(\theta)$ the set of all canonical affinor structures on a regular $\Phi$-space $G / H$. It is easy to see that $\mathcal{A}(\theta)$ is a commutative subalgebra of the algebra $\mathcal{A}$ of all invariant affinor structures on $G / H$. Moreover,

$$
\operatorname{dim} \mathcal{A}(\theta)=\operatorname{deg} \nu \leq \operatorname{dim} G / H,
$$


where $\nu$ is a minimal polynomial of the operator $\theta$. It is obvious that the algebra $\mathcal{A}(\theta)$ for any symmetric $\Phi$-space $\left(\Phi^{2}=\mathrm{id}\right)$ consists of scalar structures only, i.e., it is isomorphic to $\mathbb{R}$. As to an arbitrary regular $\Phi$-space $(G / H, \Phi)$, the algebraic structure of its commutative algebra $\mathcal{A}(\theta)$ has also been completely described (see $[10])$.

It should be mentioned that all canonical structures are, in addition, invariant with respect to the "symmetries" $\left\{S_{p}\right\}$ of $G / H$ (see [54]). Moreover, from $\left(d S_{o}\right)_{o}=\theta$ it follows that the invariant affinor structure $F_{p}=\left(d S_{p}\right)_{p}, p \in G / H$, generated by the symmetries $\left\{S_{p}\right\}$ belongs to the algebra $\mathcal{A}(\theta)$.

The most remarkable example of canonical structures is the canonical almost complex structure $J=\frac{1}{\sqrt{3}}\left(\theta^{2}-\theta\right)$ on a homogeneous 3-symmetric space (see Sec. 2). We have already seen that it is not an exception. Moreover, we are able to show that the algebra $\mathcal{A}(\theta)$ contains a rich collection of classical affinor structures.

In the sequel, we will concentrate on the following affinor structures of classical type:

almost complex structures $J\left(J^{2}=-1\right)$;

almost product structures $P\left(P^{2}=1\right)$;

$f$-structures $\left(f^{3}+f=0\right)[59]$;

$f$-structures of hyperbolic type or, briefly, $h$-structures $\left(h^{3}-h=0\right)[39]$.

Clearly, $f$-structures and $h$-structures are generalizations of structures $J$ and $P$, respectively.

All the canonical structures of classical type on regular $\Phi$-spaces were completely described $[3,14,15]$. In particular, for homogeneous $k$-symmetric spaces, precise computational formulas were indicated. For future reference, we select here some results.

Denote by $\tilde{s}(s)$ the number of all irreducible factors (respectively, all irreducible quadratic factors) over $\mathbb{R}$ of a minimal polynomial $\nu$.

Theorem 2 ([3,14,15]). Let $G / H$ be a regular $\Phi$-space.

(1) The algebra $\mathcal{A}(\theta)$ contains precisely $2^{\tilde{s}}$ structures $P$.

(2) $G / H$ admits a canonical structure $J$ if and only if $s=\tilde{s}$. In this case, $\mathcal{A}(\theta)$ contains $2^{s}$ different structures $J$.

(3) $G / H$ admits a canonical $f$-structure if and only if $s \neq 0$. In this case, $\mathcal{A}(\theta)$ contains $3^{s}-1$ different $f$-structures. Suppose that $s=\tilde{s}$. Then $2^{s} f$-structures are almost complex and the remaining $3^{s}-2^{s}-1$ have nontrivial kernels.

(4) The algebra $\mathcal{A}(\theta)$ contains $3^{\tilde{s}}$ different $h$-structures. All these structures form a (commutative) semigroup in $\mathcal{A}(\theta)$ and include a subgroup of order $2^{\tilde{s}}$ of canonical structure $P$.

Further, let $G / H$ be a homogeneous $k$-symmetric space. Then $\tilde{s}=s+1$ if $-1 \in \operatorname{spec} \theta$, and $\tilde{s}=s$ in the opposite case. We indicate explicit formulas enabling us to compute all canonical $f$-structures and $h$-structures. We shall also use the notation

$$
u= \begin{cases}n & \text { if } k=2 n+1 \\ n-1 & \text { if } k=2 n .\end{cases}
$$

Theorem 3 ([3,14,15]). Let $G / H$ be a homogeneous $\Phi$-space of order $k$.

(1) All nontrivial canonical $f$-structures on $G / H$ can be given by the operators

$$
f=\frac{2}{k} \sum_{m=1}^{u}\left(\sum_{j=1}^{u} \zeta_{j} \sin \frac{2 \pi m j}{k}\right)\left(\theta^{m}-\theta^{k-m}\right),
$$

where $\zeta_{j} \in\{-1 ; 0 ; 1\}, j=1,2, \ldots, u$, and not all coefficients $\zeta_{j}$ are zero. In particular, suppose that $-1 \notin \operatorname{spec} \theta$. Then the polynomials $f$ define canonical almost complex structures $J$ if and only if all $\zeta_{j} \in\{-1 ; 1\}$. 

(2) All canonical $h$-structures on $G / H$ can be given by the polynomials $h=\sum_{m=0}^{k-1} a_{m} \theta^{m}$, where
(a) if $k=2 n+1$, then

$$
a_{m}=a_{k-m}=\frac{2}{k} \sum_{j=1}^{u} \xi_{j} \cos \frac{2 \pi m j}{k} ;
$$

(b) if $k=2 n$, then

$$
a_{m}=a_{k-m}=\frac{1}{k}\left(2 \sum_{j=1}^{u} \xi_{j} \cos \frac{2 \pi m j}{k}+(-1)^{m} \xi_{n}\right) .
$$

Here the numbers $\xi_{j}$ take their values from the set $\{-1 ; 0 ; 1\}$ and the polynomials $h$ define canonical structures $P$ if and only if all $\xi_{j} \in\{-1 ; 1\}$.

Now we particularize the results mentioned above for homogeneous $\Phi$-spaces of orders 3,4 , and 5 only. Note that there are no fundamental obstructions to considering higher orders $k$.

Corollary $2([3,14,15])$. Let $G / H$ be a homogeneous $\Phi$-space of order 3 . There are (up to sign) only the following canonical structures of classical type on $G / H$ :

$$
J=\frac{1}{\sqrt{3}}\left(\theta-\theta^{2}\right), \quad P=1 .
$$

We noted that the existence of the structure $J$ and its properties are well known (see $[24,35,55,58])$.

Corollary $3([3,14,15])$. On a homogeneous $\Phi$-space of order 4 , there are (up to sign) the following canonical classical structures:

$$
P=\theta^{2}, \quad f=\frac{1}{2}\left(\theta-\theta^{3}\right), \quad h_{1}=\frac{1}{2}\left(1-\theta^{2}\right), \quad h_{2}=\frac{1}{2}\left(1+\theta^{2}\right) .
$$

The operators $h_{1}$ and $h_{2}$ form a pair of complementary projectors: $h_{1}+h_{2}=1, h_{1}^{2}=h_{1}, h_{2}^{2}=h_{2}$. Moreover, the following conditions are equivalent:

(1) $-1 \notin \operatorname{spec} \theta$;

(2) the structure $P$ is trivial $(P=-1)$;

(3) the $f$-structure is an almost complex structure;

(4) the structure $h_{1}$ is trivial $\left(h_{1}=1\right)$;

(5) the structure $h_{2}$ is null.

General properties of the canonical structures $P$ and $f$ on homogeneous 4-symmetric spaces were investigated in [13].

Corollary $4([3,14,15])$. There exist (up to sign) only the following canonical structures of classical type on any homogeneous $\Phi$-space of order 5 :

$$
\begin{gathered}
P=\frac{1}{\sqrt{5}}\left(\theta-\theta^{2}-\theta^{3}+\theta^{4}\right), \\
J_{1}=\alpha\left(\theta-\theta^{4}\right)-\beta\left(\theta^{2}-\theta^{3}\right), \quad J_{2}=\beta\left(\theta-\theta^{4}\right)+\alpha\left(\theta^{2}-\theta^{3}\right), \\
f_{1}=\gamma\left(\theta-\theta^{4}\right)+\delta\left(\theta^{2}-\theta^{3}\right), \quad f_{2}=\delta\left(\theta-\theta^{4}\right)-\gamma\left(\theta^{2}-\theta^{3}\right), \\
h_{1}=\frac{1}{2}(1+P), \quad h_{2}=\frac{1}{2}(1-P),
\end{gathered}
$$

where

$$
\alpha=\frac{\sqrt{5+2 \sqrt{5}}}{5}, \quad \beta=\frac{\sqrt{5-2 \sqrt{5}}}{5}, \quad \gamma=\frac{\sqrt{10+2 \sqrt{5}}}{10}, \quad \delta=\frac{\sqrt{10-2 \sqrt{5}}}{10} .
$$


Moreover, the following relations are satisfied:

$$
\begin{gathered}
J_{1} P=J_{2}, \quad f_{1} P=J_{1} h_{1}=J_{2} h_{1}=f_{1}, \quad h_{1} P=h_{1}, \quad h_{2} P=-h_{2}, \\
f_{2} P=J_{2} h_{2}=-J_{1} h_{2}=-f_{2}, \quad f_{1} f_{2}=h_{1} h_{2}=0, \quad h_{1}+h_{2}=P .
\end{gathered}
$$

In addition, the following conditions are equivalent:

(1) $\operatorname{spec} \theta$ consists of two elements;

(2) the structure $P$ is trivial;

(3) the structures $J_{1}$ and $J_{2}$ coincide (up to sign);

(4) one of the structures $f_{1}$ and $f_{2}$ is null, while the other is an almost complex structure coinciding with one of the structures $J_{1}$ and $J_{2}$;

(5) one of the structures $h_{1}$ and $h_{2}$ is trivial, while the other is null.

We note that the canonical structure $P$ on homogeneous 5 -symmetric spaces was first introduced and studied in [12]. Other canonical structures on these spaces were later studied in [18-20].

It should also be mentioned that in the particular case of homogeneous $\Phi$-spaces of any odd order $k=2 n+1$ the method of constructing invariant almost complex structures was described in [44]. It can be easily seen that all these structures are canonical in the above sense.

\section{Special Canonical $f$-Structures}

In the sequel, we will mainly concentrate on special canonical $f$-structures on homogeneous regular $\Phi$-spaces.

Recall that an $f$-structure on a manifold $M$ is known to be a field of endomorphisms $f$ acting on its tangent bundle and satisfying the condition $f^{3}+f=0$ (see [59]). The number $r=\operatorname{dim} \operatorname{Im} f$ is constant at any point of $M$ (see [56]) and called a rank of the $f$-structure. Moreover, the number $\operatorname{dim} \operatorname{Ker} f=\operatorname{dim} M-r$ is usually said to be a deficiency of the $f$-structure and denoted by $\operatorname{def} f$. It is easy to see that the particular cases def $f=0$ and $\operatorname{def} f=1$ of $f$-structures lead to almost complex structures and almost contact structures, respectively.

Let $M$ be an $f$-manifold. Then $\mathfrak{X}(M)=\mathcal{L} \oplus \mathcal{M}$, where $\mathcal{L}=\operatorname{Im} f$ and $\mathcal{M}=\operatorname{Ker} f$ are mutually complementary distributions, which are usually called the first and second fundamental distributions of the $f$-structure respectively. Obviously, the endomorphisms $l=-f^{2}$ and $m=\mathrm{id}+f^{2}$ are mutually complementary projections on the distributions $\mathcal{L}$ and $\mathcal{M}$, respectively. We note that the restriction $F$ of the $f$-structure to $\mathcal{L}$ is an almost complex structure, i.e., $F^{2}=-$ id.

Let $G / H$ be a reductive homogeneous space and $\mathfrak{g}=\mathfrak{h} \oplus \mathfrak{m}$ the corresponding reductive decomposition of the Lie algebra $\mathfrak{g}$. Any invariant $f$-structure on $G / H$ determines the decomposition $\mathfrak{m}=\mathfrak{m}_{1} \oplus \mathfrak{m}_{2}$ such that the subspaces $\mathfrak{m}_{1}=\operatorname{Im} f$ and $\mathfrak{m}_{2}=\operatorname{Ker} f$ completely define the first and second fundamental distributions, respectively.

Let us turn to canonical $f$-structures on homogeneous regular $\Phi$-spaces.

Theorem 4. Let $G / H$ be a regular $\Phi$-space, $\mathfrak{g}=\mathfrak{h} \oplus \mathfrak{m}$ be the canonical reductive decomposition, $f$ be a canonical $f$-structure on $G / H$, and $\mathfrak{m}=\mathfrak{m}_{1} \oplus \mathfrak{m}_{2}$ be the decomposition of $\mathfrak{m}$ with respect to the $f$-structure. Suppose that the $f$-structure satisfies the condition

$$
f^{2}= \pm \theta f
$$

Then

$$
\left[\mathfrak{m}_{1}, \mathfrak{m}_{1}\right] \subset \mathfrak{m}_{2} \oplus \mathfrak{h} .
$$

Proof. Note that condition (4) is equivalent to

$$
f=\mp \theta f^{2} .
$$

Using (4) and (6), for any $X, Y \in \mathfrak{m}$ we obtain

$$
f\left([f X, f Y]_{\mathfrak{m}}\right)=f\left(\left[\theta f^{2} X, \theta f^{2} Y\right]_{\mathfrak{m}}\right)=(f \theta)\left(\left[f^{2} X, f^{2} Y\right]_{\mathfrak{m}}\right)=f^{2}\left(\left[f^{2} X, f^{2} Y\right]_{\mathfrak{m}}\right) .
$$


On the other hand, using (4) and (6) again, we obtain

$$
f^{2}\left(\left[f^{2} X, f^{2} Y\right]_{\mathfrak{m}}\right)=f^{2}\left([\theta f X, \theta f Y]_{\mathfrak{m}}\right)=\left(f^{2} \theta\right)\left([f X, f Y]_{\mathfrak{m}}\right)=-f\left([f X, f Y]_{\mathfrak{m}}\right) .
$$

Comparing these two formulas, we conclude that

$$
f\left([f X, f Y]_{\mathfrak{m}}\right)=0
$$

for any $X, Y \in \mathfrak{m}$. This condition is obviously equivalent to (5).

It should be mentioned that some homogeneous $k$-symmetric spaces admit canonical $f$-structures satisfying relation (5). More exactly, we have the following corollary.

Corollary 5. Let $G / H$ be a homogeneous $\Phi$-space of order $k=4 n, n \geq 1$, such that $\{i,-i\} \subset \operatorname{spec} \theta$. Then for a nontrivial canonical $f$-structure on $G / H$ satisfying condition (4) we have $\left[\mathfrak{m}_{1}, \mathfrak{m}_{1}\right] \subset \mathfrak{m}_{2} \oplus \mathfrak{h}$.

Proof. Indeed, the procedure of describing canonical structures on homogeneous $k$-symmetric spaces is constructive (see [15]). Using it, we presented the canonical $f$-structure under the required condition in [8]. It remains to apply Theorem 4.

In particular, the canonical $f$-structure on homogeneous 4-symmetric spaces satisfies condition (5). This result was first obtained in [13]. In the case $k=8$, the corresponding canonical $f$-structure can be presented in the form (see $[8,11]$ )

$$
f=\frac{1}{4}\left(\theta-\theta^{3}+\theta^{5}-\theta^{7}\right) .
$$

By Theorem 4, it also satisfies condition (5).

We also note that the inverse of Theorem 4 is not true. As a general example, for both the canonical $f$-structures on homogeneous 5-symmetric spaces (see Corollary 4 and [18-20]) condition (5) is valid. However, condition (4) for these $f$-structures is not satisfied.

\section{Special Canonical $f$-Structures in Generalized Hermitian Geometry}

Here we illustrate the role of the special canonical $f$-structures considered above in the generalized Hermitian geometry. Specifically, we present new classes of invariant Hermitian $f$-manifolds by means of the canonical $f$-structures satisfying condition (4).

First, we recall some basic notions in the generalized Hermitian geometry. The concept of the generalized Hermitian geometry created in the 1980s (see, e.g., $[38,39,41]$ ) was a natural consequence of the development of Hermitian geometry and the theory of almost contact metric structures with many applications. One of its central objects is the metric $f$-structures of classical type, which include the class of almost Hermitian structures.

Recall that an $f$-structure on a (pseudo)Riemannian manifold $(M, g=\langle\cdot, \cdot\rangle)$ is called a metric $f$-structure if $\langle f X, Y\rangle+\langle X, f Y\rangle=0, X, Y \in \mathfrak{X}(M)$ (see [39]). In this case, the triple $(M, g, f)$ is called a metric $f$-manifold. It is clear that the tensor field $\Omega(X, Y)=\langle X, f Y\rangle$ is skew-symmetric, i.e., $\Omega$ is a 2 -form on $M . \Omega$ is called a fundamental form of a metric $f$-structure $[38,39]$. It is easy to see that the particular cases def $f=0$ and $\operatorname{def} f=1$ of metric $f$-structures lead to almost Hermitian structures and almost contact metric structures, respectively.

Let $M$ be a metric $f$-manifold. Then the first and second fundamental distributions $\mathcal{L}=\operatorname{Im} f$ and $\mathcal{M}=\operatorname{Ker} f$ are mutually orthogonal. We note that in the case where the restriction of $g$ to $\mathcal{L}$ is nondegenerate, the restriction $(F, g)$ of a metric $f$-structure to $\mathcal{L}$ is an almost Hermitian structure, i.e., $F^{2}=-\mathrm{id},\langle F X, F Y\rangle=\langle X, Y\rangle, X, Y \in \mathcal{L}$.

A fundamental role in the geometry of metric $f$-manifolds is played by the composition tensor $T$, which was explicitly evaluated in [38]:

$$
T(X, Y)=\frac{1}{4} f\left(\nabla_{f X}(f) f Y-\nabla_{f^{2} X}(f) f^{2} Y\right),
$$

where $\nabla$ is the Levi-Civita connection of a (pseudo)Riemannian manifold $(M, g), X, Y \in \mathfrak{X}(M)$. 
Using this tensor $T$, the algebraic structure of a so-called adjoint $Q$-algebra in $\mathfrak{X}(M)$ can be defined by the formula

$$
X * Y=T(X, Y) .
$$

It gives the opportunity to introduce some classes of metric $f$-structures in terms of natural properties of the adjoint $Q$-algebra (see $[38,39]$ ).

We enumerate below the main classes of metric $f$-structures together with their defining properties.

$\begin{array}{lll}\text { Kf } & \text { Kähler } f \text {-structure: } & \nabla f=0 ; \\ \mathbf{H f} & \text { Hermitian } f \text {-structure: } & T(X, Y)=0, \text { i.e., } \mathfrak{X}(M) \text { is } \\ & & \text { an Abelian } Q \text {-algebra; } \\ \mathbf{G}_{\mathbf{1}} \mathbf{f} & f \text {-structure of class } G_{1}, \text { or } G_{1} f \text {-structure: } & T(X, X)=0 \text {, i.e., } \mathfrak{X}(M) \text { is } \\ & & \text { an anticommutative } Q \text {-algebra; } \\ \text { QKf } & \text { quasi-Kähler } f \text {-structure: } & \nabla_{X} f+T_{X} f=0 ; \\ \text { Kill f } & \text { Killing } f \text {-structure: } & \nabla_{X}(f) X=0 ; \\ \text { NKf } & \text { nearly Kähler } f \text {-structure, or } N K f \text {-structure: } & \nabla_{f X}(f) f X=0 .\end{array}$

The classes $\mathbf{K f}, \mathbf{H f}, \mathbf{G}_{\mathbf{1}} \mathbf{f}, \mathbf{Q K f}$ (in a more general situation) were introduced in [39] (see also [53]). Killing $f$-manifolds Kill $\mathbf{f}$ were defined and studied in $[27,28]$. The class $\mathbf{N K f}$ was first determined in [2] (see also $[7,8]$ ).

The following relationships between the classes mentioned are evident:

$$
\mathbf{K f}=\mathbf{H f} \cap \mathbf{Q K f}, \quad \mathbf{K f} \subset \mathbf{H f} \subset \mathbf{G}_{\mathbf{1}} \mathbf{f}, \quad \mathbf{K f} \subset \mathbf{K i l l} \mathbf{f} \subset \mathbf{N K f} \subset \mathbf{G}_{\mathbf{1}} \mathbf{f} .
$$

It is important to note that in the special case $f=J$ we obtain the corresponding classes of almost Hermitian structures (see [26]). In particular, for $f=J$ the classes Kill $\mathbf{f}$ and NKf coincide with the well-known class NK of nearly Kähler structures.

Now we turn to invariant metric $f$-structures on (pseudo)Riemannian homogeneous spaces.

Let $G$ be a connected Lie group, $H$ be a closed subgroup of it, and $g$ be an invariant (pseudo)Riemannian metric on the homogeneous space $G / H$. As usual, denote by $\mathfrak{g}$ and $\mathfrak{h}$ the Lie algebras corresponding to $G$ and $H$, respectively. Assume that $G / H$ is a reductive homogeneous space and $\mathfrak{g}=\mathfrak{h} \oplus \mathfrak{m}$ is the reductive decomposition of the Lie algebra $\mathfrak{g}$. We also identify $\mathfrak{m}$ with the tangent space $T_{o}(G / H)$ at the point $o=H$. Then the invariant metric $g$ is completely defined by its value at the point $o$. For convenience, we denote in the same manner both any invariant metric $g$ on $G / H$ and its value at $o$.

Recall that $(G / H, g)$ is naturally reductive with respect to a reductive decomposition $\mathfrak{g}=\mathfrak{h} \oplus \mathfrak{m}[43]$ if

$$
g\left([X, Y]_{\mathfrak{m}}, Z\right)=g\left(X,[Y, Z]_{\mathfrak{m}}\right)
$$

for all $X, Y, Z \in \mathfrak{m}$. Here the subscript $\mathfrak{m}$ denotes the projection of $\mathfrak{g}$ onto $\mathfrak{m}$ with respect to the reductive decomposition.

Any invariant metric $f$-structure on a reductive homogeneous space $G / H$ determines the orthogonal decomposition $\mathfrak{m}=\mathfrak{m}_{1} \oplus \mathfrak{m}_{2}$ such that $\mathfrak{m}_{1}=\operatorname{Im} f$ and $\mathfrak{m}_{2}=\operatorname{Ker} f$.

As was already noted (see the Introduction), the main classes of almost Hermitian structures were provided with a remarkable set of invariant examples. It turns out that there is also a wealth of invariant examples for the basic classes of metric $f$-structures. These invariant metric $f$-structures can be realized on homogeneous $k$-symmetric spaces with canonical $f$-structures. We select here some results in this direction. More detailed information can be found in [2,4-9,20,47].

Theorem 5 ([6]). Any invariant metric $f$-structure on a naturally reductive space $(G / H, g)$ is a $G_{1} f$-structure.

As a special case $(\operatorname{Ker} f=0)$, the result from [1] on almost Hermitian $G_{1}$-structures follows.

Theorem $6([6])$. Let $(G / H, g, f)$ be a naturally reductive space with an invariant metric $f$-structure that satisfies the condition $\left[\mathfrak{m}_{1}, \mathfrak{m}_{1}\right] \subset \mathfrak{m}_{2} \oplus \mathfrak{h}$. Then $G / H$ is a Hermitian $f$-manifold. 
We note that Theorem 6 is also valid for arbitrary invariant (pseudo)Riemannian metric $g$ compatible with an invariant $f$-structure on a reductive homogeneous space $G / H$ (see [16]).

Theorems 5 and 6 can be effectively provided with a large class of examples. In particular, for a semi-simple group $G$, the invariant (pseudo)Riemannian metric $g$ generated by the Killing form on any regular $\Phi$-space $G / H$ is naturally reductive with respect to the canonical reductive decomposition $\mathfrak{g}=\mathfrak{h} \oplus \mathfrak{m}$ (see [54]). Moreover, all canonical structures $f$ and $J$ on homogeneous, naturally reductive, $k$-symmetric spaces are compatible with such a metric, i.e., $f$ is a metric $f$-structure and $J$ is an almost Hermitian structure (see $[2,11])$. In what follows, by a naturally reductive decomposition we mean the canonical reductive decomposition for a regular $\Phi$-space $G / H$.

By Theorem 5, it follows (see [6]) that any canonical metric $f$-structure on a naturally reductive $k$-symmetric space $(G / H, g)$ is a $G_{1} f$-structure, and any canonical almost Hermitian structure $J$ is a $G_{1}$-structure. It gives a wealth of invariant examples of the structures of such a kind for the generalized Hermitian geometry, in particular, for Hermitian geometry.

We also mention the following relevant result.

Theorem 7 ([7,8]). Let $G / H$ be a regular $\Phi$-space, $g$ be a naturally reductive metric on $G / H$ with respect to the canonical reductive decomposition $\mathfrak{g}=\mathfrak{h} \oplus \mathfrak{m}$, and $f$ be a metric canonical $f$-structure on $G / H$. Assume that the $f$-structure satisfies the condition $f^{2}= \pm \theta f$. Then $(G / H, g, f)$ is a nearly Kähler $f$-manifold.

Now we prove the following theorem.

Theorem 8. Let $G / H$ be a homogeneous regular $\Phi$-space, $g$ be an arbitrary invariant (pseudo)Riemannian metric, and $f$ be a metric (with respect to $g$ ) canonical $f$-structure on $G / H$. If the $f$-structure satisfies the condition $f^{2}= \pm \theta f$, then $(G / H, g, f)$ is a Hermitian $f$-manifold.

Proof. By Theorem 4, condition (4) implies that of (5). Now it remains to apply [16, Theorem 3] (see comments after Theorem 6).

As an immediate application of this theorem and Corollary 5, we have the following corollary.

Corollary 6. Let $G / H$ be a homogeneous $\Phi$-space of order $k=4 n, n \geq 1$, such that $\{i,-i\} \subset \operatorname{spec} \theta$. Consider a nontrivial canonical $f$-structure on $G / H$ satisfying the condition $f^{2}= \pm \theta f$ and endowed with any invariant compatible (pseudo)Riemannian metric $g$. Then $(f, g)$ is an invariant Hermitian $f$-structure on $G / H$.

We select one particular case of the situation above as an example of general character.

Corollary 7. The canonical f-structure

$$
f=\frac{1}{4}\left(\theta-\theta^{3}+\theta^{5}-\theta^{7}\right)
$$

on a homogeneous 8-symmetric space $G / H$ endowed with any invariant compatible (pseudo)Riemannian metric $g$ is an invariant Hermitian $f$-structure.

For example, any flag manifold $\mathrm{SU}(n) / \mathrm{T}_{\max }\left(n \geq 3, \mathrm{~T}_{\max }\right.$ is a maximal torus) can be considered as a homogeneous $k$-symmetric space for any $k \geq n$ (see [32]). Hence these flag manifolds provide a particular class of homogeneous Hermitian $f$-manifolds. Specifically, we may choose $\mathrm{SU}(8) / \mathrm{T}^{7}$ as a homogeneous 8 -symmetric space.

It should be mentioned that the result of Corollary 6 for $k=4$ was already obtained in [6] (for a naturally reductive metric) and [16] (for the general case). We also note that Riemannian 4-symmetric spaces of classical compact Lie groups were classified in [31]. Therefore, this gives a long list of homogeneous Hermitian $f$-manifolds of semisimple type, whose $f$-structure is generally nonintegrable. For homogeneous spaces of solvable type, the 6-dimensional generalized Heisenberg group $(N, g)$ represented as a Riemannian homogeneous 4 -symmetric space (see [57]) is a homogeneous Hermitian $f$-manifold with 
respect to the canonical $f$-structure $f=\frac{1}{2}\left(\theta-\theta^{3}\right)$ (see $[6]$ ), where the Riemannian metric $g$ is not naturally reductive.

\section{REFERENCES}

1. E. Abbena and S. Garbiero, "Almost Hermitian homogeneous manifolds and Lie groups," Nihonkai Math. J., 4, 1-15 (1993).

2. V. V. Balashchenko, Riemannian Geometry of Canonical Structures on Regular $\Phi$-Spaces, Preprint No. 174/1994, Fakultät für Mathematik der Ruhr-Universität Bochum (1994).

3. V. V. Balashchenko, "Canonical $f$-structures of hyperbolic type on regular $\Phi$-spaces," Russ. Math. Surv., 53, No. 4, 861-863 (1998).

4. V. V. Balashchenko, "Naturally reductive Killing $f$-manifolds," Russ. Math. Surv., 54, No. 3, 623-625 (1999).

5. V. V. Balashchenko, "Extending an idea of A. Gray: Homogeneous $k$-symmetric spaces and generalized Hermitian geometry," in: Int. Congr. on Differential Geometry in Memory of Alfred Gray, September 18-23, 2000, Bilbao (Spain), Abstracts, pp. 5-7.

6. V. V. Balashchenko, "Homogeneous Hermitian f-manifolds," Russ. Math. Surv., 56, No. 3, 575-577 (2001).

7. V. V. Balashchenko, "Homogeneous nearly Kähler $f$-manifolds," Dokl. Math., 63, No. 1, 56-58 (2001).

8. V. V. Balashchenko, "Invariant nearly Kähler $f$-structures on homogeneous spaces," in: M. Fernández et al. (eds.), Global Differential Geometry: The Mathematical Legacy of Alfred Gray. Proc. of the Int. Congr. on Differential Geometry Held in Memory of Prof. Alfred Gray, Bilbao, Spain, September 18-23, 2000, Amer. Math. Soc., Providence (2001), Contemp. Math., Vol. 288, pp. 263-267.

9. V. V. Balashchenko, Invariant Nearly Kähler $f$-Structures on Homogeneous Spaces, SFB 288 Preprint No. 499, Berlin (2001).

10. V. V. Balashchenko, "The algebra of canonical affinor structures and classes of regular $\Phi$-spaces," Dokl. Math., 66, No. 1, 111-114 (2002).

11. V. V. Balashchenko, "Invariant structures generated by Lie group automorphisms on homogeneous spaces," in: N. Bokan, M. Djoric, A. T. Fomenko, Z. Rakic, and J. Wess, eds., Proc. of the Workshop "Contemporary Geometry and Related Topics" (Belgrade, Yugoslavia, 15-21 May, 2002), World Scientific (2004), pp. 1-32.

12. V. V. Balashchenko and Yu. D. Churbanov, "Invariant structures on homogeneous $\Phi$-spaces of order 5," Russ. Math. Surv., 45, No. 1, 195-197 (1990).

13. V. V. Balashchenko and O. V. Dashevich, "Geometry of canonical structures on homogeneous Ф-spaces of order 4," Russ. Math. Surv., 49, No. 4, 149-150 (1994).

14. V. V. Balashchenko and N. A. Stepanov, "Canonical affinor structures on regular $\Phi$-spaces," Russ. Math. Surv., 46, No. 1, 247-248 (1991).

15. V. V. Balashchenko and N. A. Stepanov, "Canonical affinor structures of classical type on regular $\Phi$-spaces," Sb. Math., 186, No. 11, 1551-1580 (1995).

16. V. V. Balashchenko and D. V. Vylegzhanin, "Generalized Hermitian geometry on homogeneous $\Phi$-spaces of finite order," Russ. Math., No. 10, 33-44 (2004).

17. H. Baum, T. Friedrich, R. Grunewald, and I. Kath, Twistor and Killing Spinors on Riemannian Manifolds, Teubner-Texte zur Mathematik, Vol. 124, Teubner, Stuttgart (1991).

18. Yu. D. Churbanov, "On some classes of homogeneous $\Phi$-spaces of order 5," Russ. Math., No. 2, 88-90 (1992).

19. Yu. D. Churbanov, "Canonical $f$-structures of homogeneous $\Phi$-spaces of order 5," Vestn. BGU. Ser. 1 Fiz., Mat., Mekh., No. 1, 51-54 (1994).

20. Yu. D. Churbanov, "The geometry of homogeneous $\Phi$-spaces of order 5," Russ. Math., No. 5, 68-78 (2002).

21. A. S. Fedenko, Spaces with Symmetries [in Russian], Belarusian State University, Minsk (1977). 
22. S. Garbiero and L. Vanhecke, "A characterization of locally 3-symmetric spaces," Riv. Mat. Univ. Parma (5), 2, 331-335 (1993).

23. A. Gray, "Nearly Kähler manifolds," J. Differential Geom., 4, No. 3, 283-309 (1970).

24. A. Gray, "Riemannian manifolds with geodesic symmetries of order 3," J. Differential Geom., 7, No. 3-4, 343-369 (1972).

25. A. Gray, "Homogeneous almost Hermitian manifolds," in: Proc. of the Conf. on Differential Geometry on Homogeneous Spaces, Turin, Italy, 1983, Rend. Sem. Mat. Univ. Politec. Torino, Special Issue (1983), pp. $17-58$.

26. A. Gray and L. M. Hervella, "The sixteen classes of almost Hermitian manifolds and their linear invariants," Ann. Mat. Pura Appl., 123, No. 4, 35-58 (1980).

27. A. S. Gritsans, "On the geometry of Killing f-manifolds," Russ. Math. Surv., 45, No. 4, 168-169 (1990).

28. A. S. Gritsans, "On the structure of Killing f-manifolds," Russ. Math., 36, No. 6, $46-54$ (1992).

29. R. Grunewald, "Six-dimensional Riemannian manifolds with a real Killing spinor," Ann. Global Anal. Geom., 8, No. 1, 43-59 (1990).

30. S. Helgason, Differential Geometry, Lie Groups, and Symmetric Spaces, Academic Press, New York (1978).

31. J. A. Jimenez, "Riemannian 4-symmetric spaces," Trans. Amer. Math. Soc., 306, No. 2, 715-734 (1988).

32. J. A. Jimenez, "Existence of Hermitian $n$-symmetric spaces and of non-commutative naturally reductive spaces," Math. Z., 196, No. 2, 133-139 (1987).

33. I. Kath, "Pseudo-Riemannian T-duals of compact Riemannian homogeneous spaces," Transform. Groups, 5, No. 2, 157-179 (2000).

34. V. F. Kirichenko, "K-spaces of constant type," Sib. Math. J., 17, 220-225 (1976).

35. V. F. Kirichenko, "On the geometry of homogeneous K-spaces," Math. Notes, 30, 779-785 (1981).

36. V. F. Kirichenko, "Sur la geometrie des varietes approximativement cosymplectiques," C. R. Acad. Sci. Paris, Sér. 1, 295, No. 12, 673-676 (1982).

37. V. F. Kirichenko, "Hermitian-homogeneous generalized almost Hermitian manifolds," Sov. Math. Dokl., 30, 267-271 (1984).

38. V. F. Kirichenko, "Quasi-homogeneous manifolds and generalized almost Hermitian structures," Math. USSR Izv., 23, 473-486 (1984).

39. V. F. Kirichenko, "Methods of generalized Hermitian geometry in the theory of almost contact manifolds," J. Sov. Math., 42, No. 5, 1885-1919 (1988).

40. V. F. Kirichenko, "Generalized quasi-Kählerian manifolds and axioms of $C R$-submanifolds in generalized Hermitian geometry. I," Geom. Dedicata, 51, 75-104 (1994).

41. V. F. Kirichenko, Differential-Geometric Structures on Manifolds [in Russian], MPGU, Moscow (2003).

42. V. F. Kirichenko and L. V. Lipagina, "Killing $f$-manifolds of constant type," Izv. Math., 63, No. 5, 963-981 (1999).

43. S. Kobayashi and K. Nomizu, Foundations of Differential Geometry, Vol. 2, Interscience-Wiley, New York (1969).

44. O. Kowalski, Generalized Symmetric Spaces, Lect. Notes Math., Vol. 805, Springer, Berlin (1980).

45. S. Lang, Algebra, Addison-Wesley Ser. Math., Addison-Wesley (1965).

46. A. J. Ledger and L. Vanhecke, "On a theorem of Kiričenko relating to 3-symmetric spaces," Riv. Mat. Univ. Parma (4), 13, 367-372 (1987).

47. L. V. Lipagina, "On the structure of the algebra of invariant affinor structures on the sphere $S^{5}$," Russ. Math., 41, No. 9, 15-18 (1997).

48. S. Salamon, "Harmonic and holomorphic maps," in: Geometry Seminar "Luigi Bianchi" II - 1984, Lect. Notes Math., Vol. 1164, Springer, Berlin (1985), pp. 161-224. 
49. S. M. Salamon, "Minimal surfaces and symmetric spaces," in: Differential Geometry. Proc. 5th Int. Colloq., Santiago de Compostela/Spain, 1984, Res. Notes Math., 131, 103-114 (1985).

50. T. Sato, "Riemannian 3-symmetric spaces and homogeneous K-spaces," Mem. Fac. Technology, Kanazawa Univ., 12, No. 2, 137-143 (1979).

51. K. Sekigawa and J. Watanabe, "On some compact Riemannian 3-symmetric spaces," Sci. Rep. Niigata Univ. Ser. A, No. 19, 1-17 (1983).

52. K. Sekigawa and H. Yoshida, "Riemannian 3-symmetric spaces defined by some outer automorphisms of compact Lie groups," Tensor, 40, No. 3, 261-268 (1983).

53. K. D. Singh and R. Singh, "Some $f(3, \varepsilon)$-structure manifolds," Demonstratio Math., 10, No. 3-4, 637-645 (1977).

54. N. A. Stepanov, "Basic facts of the theory of $\varphi$-spaces," Izv. Vyssh. Uchebn. Zaved., Mat., No. 3, 88-95 (1967).

55. N. A. Stepanov, "Homogeneous 3-cyclic spaces," Izv. Vyssh. Uchebn. Zaved., Mat., No. 12, 65-74 (1967).

56. R. E. Stong, "The rank of an f-structure," Kōdai Math. Sem. Rep., 29, 207-209 (1977).

57. F. Tricerri and L. Vanhecke, Homogeneous Structures on Riemannian Manifolds, London Math. Soc. Lect. Note Ser., Vol. 83, Cambridge Univ. Press (1983).

58. J. A. Wolf and A. Gray, "Homogeneous spaces defined by Lie group automorphisms," J. Differential Geom., 2, No. 1-2, 77-159 (1968).

59. K. Yano, "On a structure defined by a tensor field $f$ of type $(1,1)$ satisfying $f^{3}+f=0$," Tensor, 14, 99-109 (1963).

Vitaly V. Balashchenko

Faculty of Mathematics and Mechanics, Belarusian State University,

F. Scorina av. 4, Minsk 220050, Belarus

E-mail: balashchenko@bsu.by, vitbal@tut.by 